\title{
An Introductory Editorial: Special Issue on Global Wireless Summit (GWS-2015)
}

\author{
Ashok Chandra ${ }^{1} \cdot$ Neeli R. Prasad ${ }^{2}$
}

Published online: 29 August 2017

(C) Springer Science+Business Media, LLC 2017

The last decade has been witnessing fastest developments in wireless communications and ICT technologies worldwide particularly new wireless based systems. The Wireless communications, besides providing the last mile access and the vital mobility, revolutionized the way we communicate, stay connected, learn and even entertained. This has affected our day to day life with lot of aspirations of providing ubiquitous and seamless connectivity. Pioneering work and developments in communication systems, protocols and VLSI technology played a catalytic role in making a quick evolution possible. The yet other area of ICT which is going to revolutionize the way we communicate with and handle things including gadgets and machines is Internet of Things (IoT). These areas have been experiencing vibrant research and technology development activities. The Global Wireless Summit (GWS) was conceptualized in bringing together wireless media communication/ personal communication, etc. The First GWS was successfully held in Princeton (USA), in 2013, followed by the Second one in Aalborg (Denmark), in 2014.

The 3rd in series of the Global Wireless Summit, the GWS-2015 organized two major conferences namely 18th International Symposium on Wireless Personal Communications (WPMC) and 5th Wireless VITAE, which was held in Hyderabad (India) during December 13-16, 2015. The first day i.e. December 13, 2015 was devoted to Tutorial sessions by leading experts. The focus for 3 days (December 14-16) was also on green and secure communication, wherein government, academia, industry and standardization bodies actively participated. The GWS-2015 was on the theme of 'Democratizing Communications'. In GWS-2015, more than 200 thoroughly reviewed papers, were presented, from all over the world.

Ashok Chandra

drashokchandra@gmail.com

1 Ministry of Communications and Information Technology (Former), New Delhi, India

2 SPA Solutions - LLC, Mount View, CA, USA 
An announcement was made by the Organizing Committee of GWS-2015, that a Special Issue in an internationally reputed Journal will be launched to cover high quality papers. As a follow up, 50+ high ranking papers were short-listed amongst the papers presented at GWS-15 and the authors were invited to submit the extended version of their papers for consideration for publication in a Special Issue of the International Journal of Wireless Personal Communications (WPC) on GWS-15. The authors were advised to maintain the quality and integrity with significant contributions and should not have any plagiarized content from any source, even from own work.

The topics covered were Wireless Communications and Physical Layer; Internet of Things; Machine to Machine (M2M) Communications; Wireless Networks; Communications Services and Multimedia Applications; 5G; WLAN; WPAN; Radio Resource Management; and Optical Wireless; etc. The extended version of these papers was again reviewed by the experts and finally 27 papers have been published in this series of WPC on GWS-2015.

We, mention about the papers below for quick reference of the readers. The papers are broadly categorized under five groups namely Algorithms and Channel Estimation in Wireless Communications; Antenna and circuit design; Opportunistic Approaches in Communication Technologies; Special interest; and Vehicular \& Mobile Ad hoc Networks and Wireless Sensor Networks.

\section{Algorithms and Channel Estimation in Wireless Communications}

1. "New Algorithm for Time and Frequency Synchronization in MIMO-OFDM Systems" by Sandeep Kumar Singh, Anagha P. Rathkanthiwar, and Abhay S. Gandhi;

2. "Dynamic Analysis of Load Balancing Algorithms in LTE/LTE-A HetNets" by Diego Castro-Hernandez, and Raman Paranjape;

3. Performance Evaluation of Novel Microwave Imaging Algorithms for Stroke Detection using an Accurate 3D Head Model" by Elisa Ricci, Ernestina Cianca, Tommaso Rossi, arina Diomedi, and Parth;

4. "Interference Analysis and Mitigation Techniques in Wireless Body Area Networks" by Pradnya H. Ghare, and A. G. Kothari;

5. "A Compressive Sensing Channel Estimation for MIMO FBMC/OQAM System" by Meng Lin, Yunzhou Li, Limin Xiao, and Jing Wang; and

6. "Multi-Dimensional Wireless Tomography Using Tensor-Based Compressed Sensing" by Takahiro Matsuda, Kengo Yokota, Kazushi Takemoto et al.

\section{Antenna and Circuit Design}

1. "Investigations on SAR and Thermal Effects of a Body Wearable Microstrip Antenna" by Varshini Karthik and T. Rama Rao;

2. "SIW Based Antipodal Linear Tapered Slot Antenna for Inter-Satellite Communication Links at $60 \mathrm{GHz}$ " by T. Rama Rao and Nishesh Tiwari;

3. "Design and Performance Analysis of a Penta-band Spiral Antenna for Vehicular Communications" by Ramya and T. Rama Rao; and

4. "UWB Sensing Antenna, Reconfigurable Transceiver and Reconfigurable Antenna Based Cognitive Radio Test Bed” by Sumin D. Joseph, S. Manoj, Chetan Waghmare, K. Nandakumar, and Ashwin Kothari. 


\section{Opportunistic Approaches in Communication Technologies}

1. "Participation of Optical Backbone Network in Successful Advancement of Wireless Network" by Rajarshi Mahapatra;

2. "An Efficient Visibility Prediction Framework for Free-Space Optical Systems" by Ivo Sousa, Maria Paula Queluz, and António Rodrigues;

3. "Spectrum Efficiency at the Extensive and Intensive Edges" by Benoit Pierre Freyensl;

4. "Inter-Cloud Communication Through Gatekeepers to Support IoT Service Interaction in the Arrowhead Framework" by Pál Varga, and Csaba Hegedüs;

5. "An Incentive-Based Scheme for Mitigating Node Selfishness in Smart Opportunistic Mobile Networks" by C. C. Sobin, Vaskar Raychoudhury, and Snehanshu Saha; and

6. "Performance Analysis of Hybrid Duplex Cellular System Using Frequency Reuse" by Wenping Bi, Xin Su, Limin Xiao, and Shidong Zhou.

\section{Special Interest}

1. "Secure Persuasive Business Models and Business Model Innovation in a World of 5G" by Peter Lindgren, and Katharina Wuropulos;

2. "Chaotic Visual Cryptography Based Digitized Document Authentication" by Anirban Goswami, Ritesh Mukherjee, and Nabin Ghoshal;

3. "Dynamic Authentication Protocol Using Multiple Signatures" by Soumit Chowdhury, Ritesh Mukherjee and Nabin Ghoshal;

4. "Recognising Daily Functioning Activities in Smart Homes" by Aristodemos Pnevmatikakis; and

5. "Chronic Wound Image Analysis by Particle Swarm Optimization Technique for Tele-Wound Network" by Chinmay Chakraborty.

\section{Vehicular and Mobile Ad Hoc Networks and Wireless Sensor Networks}

1. "Network Coding Based Distributed Indoor Target Tracking Using Wireless Sensor Networks" by Laxminarayana S.Pillutl;

2. "Prioritized Mobile Node Deployment to Enhance the Lifetime of Wireless Sensor Network" by J. Roselin and P. Latha;

3. "Conducted EMI Analysis of Vehicle Positioning System and Strategies to Reduce Conducted EMI" by Shreenivas Jog, Nidhi Chaturvedi, and Rupa Burli;

4. "A Novel Interdependent Source-Channel Coding Technique for Enhanced Energy Efficiency in Communication over Wireless Sensor Networks" by N. C. Resmi, and Sonali Chouhan;

5. "Study of Radiated Emission from Wireless Positioning System and the Strategies to Minimize the Radiations" by Shreenivas Jog, Vatsla Bhatnagar, Rupa Burli, and Suvidha Chitnis Deshpande; and

6. "Aerial-Heterogeneous Network: A Case Study Analysis on the Network Performance Under Heavy User Accumulations" by Purnima Lala Mehta, and Ramjee Prasad. 
We are sure that this Special Issue covering wide ranging topics on wireless communications and ICT will open new doors of knowledge. The ideas enunciated will certainly be of immense use to the researchers.

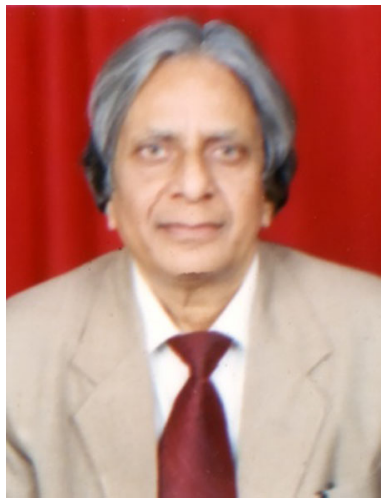

Dr. Ashok Chandra is the former Wireless Adviser to the Government of India. Presently, he is working as a Guest Scientist in the Department of Business Technology, Aarhus University, Denmark. In his responsibility as Wireless Adviser, he was associated with national spectrum management activities, including in spectrum planning and engineering, frequency assignment, frequency coordination, spectrum monitoring, policy regarding regulatory affairs for new technologies and related research \& development activities, etc. From the spectrum management points of view, he played a key role in the auction of $2 \mathrm{G}$, 3G and Broadband Wireless Access (BWA) spectrum. Dr. Chandra, an "Indian Engineering Services" officer did his Post graduate in Electronics, $\mathrm{PhD}$ and Doctorate of Science (D.Sc.) in Radio Mobile Communications. He has worked as a Guest Scientist in various Technical Universities of Germany and Denmark. Dr. Chandra is having Technical Experience of over 35 years in the field of Radio Communications/Radio Spectrum Management sector. He has published over 30 research contributions in the fields of Wireless Communications. He has about 7 years of experience dealing with Technical Education matters of Indian Institutes of Technology (IIT), Indian Institute of Science (IISc) etc. particularly their various research projects also in the areas of telematics/radio communications. He had played a key role in the establishment of new Indian Institutes of Technology, Indian Institute of Management and Indian Institutes of Information Technology. Dr. Chandra was instrumental in the implementation of "Technology Development Mission" scheme, under which one IIT/IISc was identified as a lead institution and two institutes as participating partners with the involvement of industry in each generic area. He has visited various technical Institutions and Universities namely Technical University of Aachen, Germany; Aalborg University, Denmark; Bremen University, Germany; and University of Lisbon, Portugal etc. and took several lectures in the area of radio mobile communications in these universities. He has chaired various Technical Sessions at the International Conferences. As the Project Director, he implemented a World Bank assisted Project in the Ministry of Communications \& IT, Government of India on "National Radio Spectrum Management and Monitoring System". The objectives of this system were automation of the process for Radio Frequency (RF) spectrum management and upgradation of radio monitoring facilities, with the aim for optimal and efficient usages of scarce limited natural resource of RF spectrum. He served as a Vice-Chairman, Study Group 5 of International Telecommunications Union (ITU)-Radio Sector. He has represented India to a large number of ITU meetings including World Radio Conferences (WRC). He served as Councilor from Indian Administration in ITU Council. He organized Working Party 5 D of ITU R Study Group 5 meeting in India in the year 2011. He was TPC Executive Chair of Global Wireless Summit 2015.

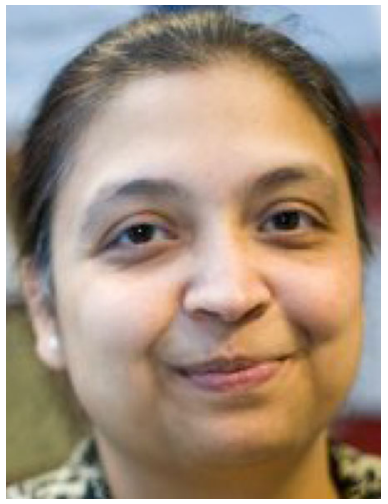

Dr. Neeli R. Prasad is the Founder CEO of the SPA solutions-LLC, Mount View, California, USA. She is the former Research Head, CTIF, Aalborg University, Denmark and Director CTIF-USA. She is a security and wireless technology strategist, who through her career has been driving business and technology innovation, from incubation to prototyping to validation. She is Master of Science degree from Delft University, Netherlands and $\mathrm{PhD}$ degree in Electrical and Electronic Engineering from University of Rome Tor Vergata, Italy. She has more than 250 publications and published two of the first books on WLAN. She is an IEEE senior member and an IEEE Communications Society Distinguished Lecturer. Dr. Prasad was the executive co-chair of IEEE GLOBECOM 2015. She has focus and the abilities to transform organizations and networking technologies to address changes in markets. She has made her way up the waves of secure communication technology by contributing to the most groundbreaking and commercial inventions. She has general management, leadership, and technology skills, having worked for service providers and technology companies in various key leadership roles. She was leading a global team of $20+$ researchers across multiple technical areas and projects in 
Japan, India, throughout Europe and USA. She has been involved in projects and plays a key role from concept to implementation to standardization. Her strong commitment to operational excellence, innovative approach to business and technological problems, and aptitude for partnering cross-functionally across the industry have reshaped and elevated her role as project coordinator making her the preferred partner in multinational and European Commission project consortium. Her notable accomplishments include enhancing the technology of multinationals including CISCO, HUAWEI, NIKSUN, Nokia-Siemens and NICT, defining the reference framework for Future Internet Assembly and being one of the early key contributors to Internet of Things. She is also expert member of governmental working groups and cross continental forums. Previously, she has served as chief system/network architect on large-scale projects from both the network operator and vendor looking across the entire product and solution portfolio covering security, wireless, mobility, Internet of Things, Machine-to-Machine, eHealth, smart cities and cloud technologies. She was one of the key contributors to the commercialization of WLAN for which she has published two books. 